\title{
Neurological complications associated with chronic Behçet's disease: Case report and review
}

\author{
Andrew Dookhan*1, Abhinav Sinha ${ }^{2}$ \\ ${ }^{1}$ Spartan Health Sciences University, Saint Lucia \\ ${ }^{2}$ Division of Medicine at North Vista Hospital, North Las Vegas, United States
}

Received: September 12, 2017

Accepted: November 6, 2017

Online Published: November 12, 2017

DOI: $10.5430 /$ crim.v4n $4 \mathrm{p} 43$

URL: https://doi.org/10.5430/crim.v4n4p43

\begin{abstract}
Behçet's disease is a multi-systemic vasculitis causing inflammation to blood vessels throughout the body. It is characterized most commonly by recurrent oral and genital ulcers but also susceptible to ocular, neurologic, vascular, articular, and gastrointestinal symptoms as well. Although etiology of this disease is still unclear and diagnosis is clinically based, there is a consistent association with the human leukocyte antigen complex, particularly HLA-B51.

This case report used the patient's chronic disease course along with various imaging studies to conclude the correlation of severe complications that may present later in life of these types of patients.

There was positive neurological involvement indicated with an acute infarct in parts of the brain hemispheres exclusively to the temporal and parietal lobes. Central nervous system involvement was more common in this patient, which manifested with noticeable unilateral weakness along with slurred speech.

Although neurologic complications within Behçet's disease are indeed rare, it is one of the most dangerous manifestations to present in regard of an acute cerebrovascular accident (CVA). This review focused on the neurocognitive complications of chronic Behçet's disease and how these patients should be closely monitored to help prevent the neurological complications that may present.
\end{abstract}

Key Words: Behçet's, Neuro-Behçet's, Neurological deficits

\section{INTRODUCTION}

Behçet's disease is a particularly rare autoimmune disorder that causes inflammation of blood vessels throughout the body. ${ }^{[1]}$ Estimates of prevalence in the United States and Europe have ranged from 0.12 to 7.5 per $100,000 .{ }^{[2]}$ This inflammatory disease characterized by recurrent oral aphthous ulcers and numerous potential systemic manifestations such as genital ulcers, skin lesions, ocular, neurologic, vascular, articular, and gastrointestinal disease. ${ }^{[3]}$
Pathophysiologic etiology is still unclear but there is a known association of an immune complex vasculitis, involving small vessels. ${ }^{[4]}$

Reports of Behçet's disease neurological involvement, ranged from 5\% to $10 \%$ in a large series. Reports on clinical and radiologic aspects of neuro-Behçet syndrome (NBS) are generally limited in number. ${ }^{[5]}$ Central nervous system involvement is one of the most threatening indications in which it tends to involve the white matter portion of the brain and

*Correspondence: Andrew Dookhan; Email: Andrew.dookhan@gmail.com; Address: Spartan Health Sciences University, Saint Lucia. 
brainstem that may lead to headaches, confusion, strokes, personality changes, and dementia rarely. ${ }^{[6]}$

Within all of the several neurological manifestations linked in association with Behçet's disease, although rare, focused assessment is placed on the aspect of a cerebrovascular accident (CVA).

\section{Case presentation}

A 51-year-old, Caucasian female, presented to the emergency department complaining of confusion and memory loss for the past 12 hours. On admission, she was disoriented and could not remember her name and "did not know what was going on". She never had a previous episode like this before. The patient experienced altered mental status with impaired cognition and memory loss, along with generalized weakness, fatigue, myalgia, diffuse lower back pain, joint pain and stiffness for the past one-month without improvement.

Review of systems noted painful bilateral lower extremity erythematous rashes with paresthesias along with painful oral and labial ulcers.

The patient's family history was negative and her past medical history is only significant for Behçet's disease, diagnosed nine years ago prior to admission via an increased HLA-B51 gene expression at a study conducted at the Cleveland clinic (2008). The course of her disease has been conservatively managed prior to admission with oral prednisone $-40 \mathrm{mg}$ daily.

On physical examination, the patient was alert and oriented $\times 1$-disoriented to name and time, acknowledged she was in a hospital. Neurologic exam presented an anxious mood, decreased concentration and fund of knowledge, aphasia, painful and decreased motor strength on lower extremities bilaterally, and reflexes: 2/4-C7, L4, S1 throughout. Other pertinent physical findings revealed erythema nodosum, numerous oropharyngeal aphthous ulcers, as well as ulcerations of the labia minora.

A clinical diagnosis of Behçet's disease exacerbation with acute encephalopathy was the initial impression made and the patient was admitted and immediately started on IV solumedrol - $500 \mathrm{mg}$ BID.

The patient's initial work-up was extensive in nature consisting of the following:

- Electrocardiogram (ECG), troponin T, troponin I, BNP

- Echocardiogram

- Electroencephalogram (EEG) - Awake/Asleep

- Comprehensive metabolic panel (CMP), magnesium, phosphorous

- Complete blood count (CBC) with differential, HbA1c
- PTT, PT, INR

- Iron panel, ferritin

- TSH, Vit. B12, Folate

- Vit. D,25-Hydroxy, LC/MS/MS

- Urinalysis; microscopic; with reflex

- Protein, urine, random

- Creatinine, urine, random

- Renal function panel

- C-reactive protein (CRP), erythrocyte sedimentation rate (ESR), Rheumatoid factor

- ANA titer

- ANCA panel

- Glomerular basement membrane AB (IgG)

- C3 complement

- C4 complement

- Anti-cardiolipin Ab, IgG, IgM, QN

- Homocysteine (cardio), FPIA

- RPR w/ RFL titer and conf

- Protein C antigen, total; deficiency panel

- Protein S panel, total

- Factor X activity

- Factor VII antigen

- Factor V Leiden

- Factor II gene mutation

All initial workup testing for various etiologies of the patient's symptoms of acute encephalopathy and vasculitis were ruled negative except for a positive urinalysis for an asymptomatic bacteriuria which was treated with oral ciprofloxacin.

The negative testing further supported the admitting diagnosis of an exacerbation of Behçet's disease from the patient's presenting altered mental status and multi-organ inflammatory symptoms.

The patient remained stable with unremarkable findings until the morning of day 3 , length of stay, where she presented with physical findings of right-sided hemiparesis and dysarthria. A neurology and cardiology consults were ordered based on physical findings which warranted the following neurological imaging testing: $:{ }^{[4]}$ CT head without contrast, MRI brain without contrast, transesophageal echocardiogram (TEE), and CT angiogram neck with and/or without contrast; accompanied with images respectively. Results noted she experienced an acute CVA. Radiologic interpretations with images are documented and correlated on the findings below.

\subsection{CT Head without contrast}

\subsubsection{Findings}

$4 \mathrm{~cm}$ wedge-shaped peripheral hypodense lesion in the left posterior superior parietal lobe. There is no mass effect. No 
hemorrhage. No subdural hematoma. No midline shift. The ventricles and sulci are normal in size.

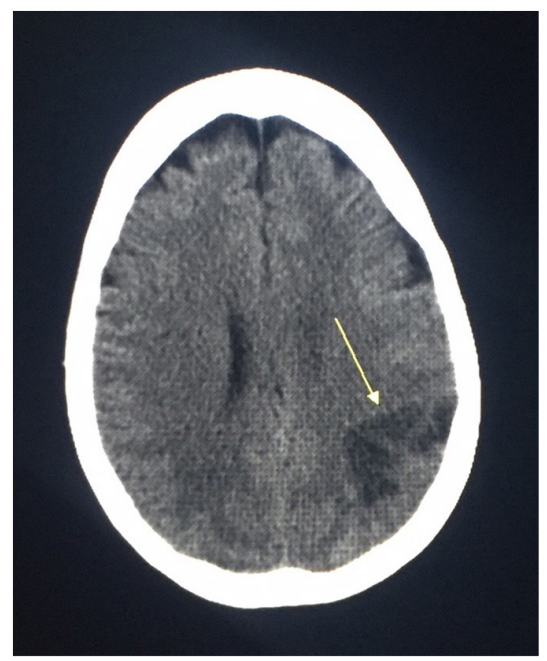

\subsubsection{Impression}

Peripheral wedge-shaped hypodense lesion in left posterior superior parietal lobe. This is a subacute infarct.

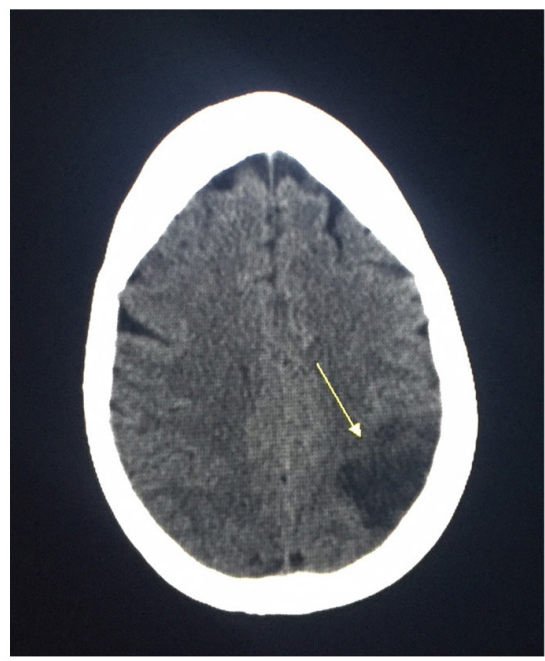

Figure 1. CT Head without contrast - thin axial images of the head

Images are in the modality of CT scan without contrast labeled as Figure 1; yellow arrow indicates the subacute infarct.

\subsection{MRI Brain without contrast}

\subsubsection{Findings}

There is restricted diffusion, hypodense lesions in the left temporal and parietal lobe indicating an acute infarct. There is an old lacuna in the left caudate head. No hemorrhage. No mass. No subdural hematoma. The thalami and the brainstem are normal in signal intensity. Normal size ventricles and sulci. No atrophy. Normal-sized pituitary and corpus callosum. No cerebellar pontine angle mass. The sinuses and mastoids are normal.

\subsubsection{Impression}

1) Acute non-hemorrhagic left temporal and parietal lobe infarct.

2) Old lacunar infarct in the left carotid head.

Images are in the modality MRI brain and labeled as Figure 2 ; viewed with different densities and contrast settings. Yellow arrow indicates the acute non-hemorrhagic temporal and parietal lobe infarct.

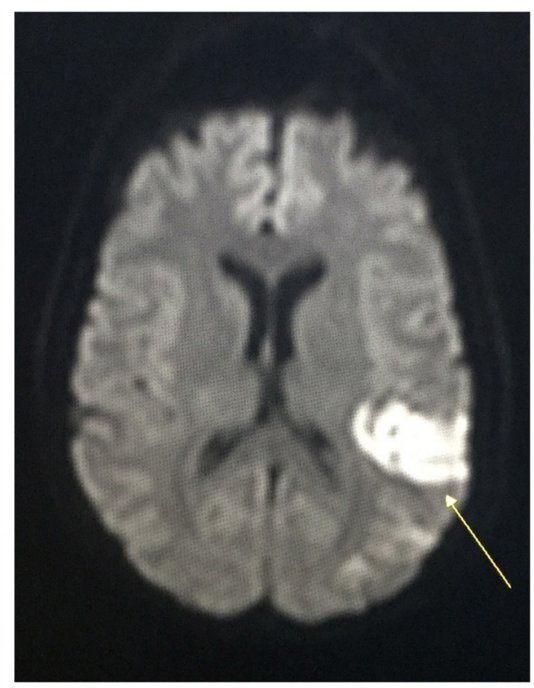

Figure 2. MRI brain without contrast - sagittal T1, axial T1, T2, FLAIR, diffusion, ADC map and gradient echo sequences (1.5 Telsa). 
3) TEE from the cardiology consult was ordered to rule out a thrombus shunt from paradoxical embolization, which revealed no vegetation's present.

4) $\mathrm{CT}$ angiogram neck with and/or without contrast resulted in negative findings and it was concluded to have no hemodynamically significant stenosis of the major intracranial and carotid arteries nor intracranial aneurysm noted.

Treatment orders consisted of the following:

- Solumedrol - 500 mg IV BID

- Aspirin EC - 325 mg PO daily

- Temovate - $0.05 \%$ ointment topically BID

- Heparin (Porcine) - 5,000 units SC Q12 hours

- MS-Contin - 15 mg PO Q12 hours

- Zofran - 4 mg IV Q6 hours or as needed

- Protonix - $40 \mathrm{mg}$ PO once every morning before breakfast

The patient continued hospitalization for an additional 4 days following the CVA. She tolerated the medications well and responded with signs of increased mental and physical competence.

Physical exam findings on day 5, revealed an improved expressive aphasia.

Discharge plan was initiated and was scheduled for after day 7 LOS.

Discharge plan includes:

- Medications - continue aspirin and change IV solumedrol to PO prednisone - $20 \mathrm{mg}$ BID

- Rehabilitation - physical therapy/occupational therapy and speech therapy at a rehabilitation facility

- Continue family support

- Follow up appointment with neurology scheduled in two weeks

\section{Discussion}

Behçet's disease is an autoimmune multi-systemic vasculitis causing inflammation to blood vessels throughout the entire body. It is most commonly characterized by recurrent oral and genital ulcers but also susceptible to ocular, neurologic, vascular, articular, and gastrointestinal symptoms as well.

The etiology of this disease is still unclear and diagnosis is usually clinically based. However, there is a consistent association with the human leukocyte antigen complex, particularly HLA-B51.

Based on this patient's presentation, deficits noted were primarily of neurologic origin in contrast to any other organ system involvement, specifically due to the severity of her hospitalization. Although there is not a vast amount of information currently on Behçet's disease, research exhibited by Borhani-Haghighi's study in 2006, stressed that central nervous system involvement was more common than peripheral nervous system manifestations with headache, motor weakness, and paresthesias were the most common presenting symptoms .

A viable discussion that may arise is that indeed there are other possible etiologies that may have attributed to this patient's acute CVA other than her primary Behcet's disease, ranging from cardiovascular to hypercoagulable hematologic disorders, including rarer causes such as immunologic vasculitides. This may allude to question what positive risk factors and pertinent test findings in this patient could have helped us to pinpoint to what precisely contributed to this patient's neurological complication.

The 51-year-old female had a negative family history and past medical history for any cardiovascular risk factors: female age $<55$ years old, no history of atrial fibrillation and/or prosthetic heart valves, non-diabetic, non-hypertensive, no hypercholesterolemia, and a lifetime nonsmoker. These negative risk factor associations along with initial workup results of the negative ECG, echocardiogram, and EEG helps solidify that the cause of the patient's CVA was not of a thrombotic etiology and not in any origin of cerebral vascular disease triggered by atherosclerotic plaques.

The patient's negative medical history, initial workup findings, along with the TEE and CT angiogram neck performed later in the patient's clinical course additionally supports that the CVA was also not of a cardiovascular embolic etiology. Respectively, no vegetation nor stenosis of intracranial and carotid arteries were found upon conclusion.

Furthermore, the results of the non-contrast CT scan and MRI brain ruled out a CVA of hemorrhagic etiology, but, neuroimaging remarkably revealed the patient suffered a $4 \mathrm{~cm}$ wedge-shaped infarct in the left posterior superior parietal lobe and multiple acute non-hemorrhagic infarcts in the left temporal and parietal lobes along with an old lacunar infarct in the left carotid head, respectively.

These findings offer supporting evidence that the CVA was embolic in nature due to the fact that it was a wedge-shaped infarct, alongside revealing an asymptomatic previous old lacunar infarct in the left carotid head that was possibly undiagnosed at an earlier stage of the patient's chronic disease.

In regards to the cerebral embolism pathogenesis, a supplementary hematologic disorder workup of coagulation cascade dysfunction (i.e.: Factor and protein C/S activity) test- 
ing resulted in negative findings and ruled out possible hypercoagulable etiologies.

Nutritional deficiencies, infectious, and metabolic disorders were ruled out as well with negative findings (i.e.: Vit. B12, folate; RPR for neurosyphilis; TSH). Numerous other immunologic vasculitis' etiologies were also excluded without any presence of inflammatory markers and antibody/antigen immunocomplexes (i.e.: CRP, ESR, RF; ANA, ANCA, C3 \& $\mathrm{C} 4$ complement, Glomerular basement membrane IgG, Anti-cardiolipin IgM/IgG, Homocysteine).

Therefore, it can be ultimately perceived through the extensive workup conducted along with a clinical diagnosis of exclusion, etiology for this patient's acute CVA in the ipsilateral temporal and parietal lobes were indeed due to the fact, although rare, a neurovascular complication of Behçet's disease.

CNS involvement of Behçet's disease can result in lesions within the cerebral hemispheres, clinically may present as aphasia, limb weakness or numbness, and brainstem complications such as difficulties with vision, hearing, or, sometimes change in mental status and respiratory arrest. ${ }^{[7]}$ These symptoms were almost entirely experienced by this patient from arrival at the emergency department up to the total LOS in the hospital.

The patient's acute CVA experienced while admitted at the hospital was one of the more common neurologic manifestations seen in a person with Behçet's disease. Compared to the findings from the 2006 study: hemispheral and brainstem stroke-like syndromes and cerebral venous thrombosis were the major neurologic presentations. ${ }^{[8]}$ MRI imaging findings included single or multiple tiny ischemic/demyelinative lesions (iso/hypodense in T1 weighted images and hyperintense in T2 images and FLAIR). ${ }^{[8]}$ This patient's radiographic MRI results: restricted diffusion, hypodense lesions in the left temporal and parietal lobe indicating an acute infarct was indeed nearly parallel with the study conducted.

In conclusion, Behçet's disease is an autoimmune multisystem inflammatory disease with a remarkable chronic course. The etiology, although unknown, vasculitis is found to be the confounding pathologic presenting feature. Most patients with Behçet's disease commonly experience recurrent oral ulcers and genital ulcers. Presentation although can vary; one detrimental complication that may occur is the presence of neurological deficits such as an acute CVA, most commonly affecting the cerebral hemispheres that may present with dysarthria and unilateral hemiparesis.

Based on the severity of this autoimmune phenomenon, healthcare professionals need to anticipate the neurologic complications that these patients may succumb to with Behçet's disease.

Prevalence and knowledge of this disease is somewhat limited; it is critical for these patients to be closely monitored medically without delay of treatment to further prevent any neurological deficit that may arise upon encounter.

\section{CONFLicts OF INTEREST Disclosure}

The authors have declared no conflicts of interest.

\section{REFERENCES}

[1] Mayo Clinic Staff. Behcet's Disease. Available from: http://www.mayoclinic.org/diseases-conditions/ behcets-disease/home/ovc-20178984

[2] Calamia KT, Wilson FC, Murat I, et al. Epidemiology and clinical characteristics of Behçet's disease in the US: A population-based study. Arthritis \& Rheumatism. 2009; 61(5): 600-4. PMid:19405011 https://doi.org/10.1002/art. 24423

[3] Smith EL. Yazici Y. Pathogenesis of Behcets syndrome. Available from: http://www.uptodate.com/contents/pathogen esis-of-behcets-syndrome?source=see_link

[4] Sattar HA. Fundamentals of Pathology, 1st edition - p. 99.
[5] Koçera N, Islaka C, Sivaa A, et al. CNS Involvement in Neuro-Behcet Syndrome: An MRI Study. American Journal of Neuroradiology. 1999 June; 20 (6): 1015-24. PMid:10445437

[6] The John Hopkins Vasculitis Center. Behcet's Disease. Available from: http://www.hopkinsvasculitis.org/types-vasculi tis/behcets-disease/\#brain

[7] Ahmad S, Alfedaghi AS, Masters Y, et al. A brain mass in a patient with Behcet's disease: A case report. Journal of Medical Case Reports. 2015; 9: 209. PMid:26419933 https://doi.org/10.118 6/s13256-015-0694-5

[8] Borhani-Haghighi A, Samangooie S, Ashjazadeh N, et al. Manifestation of Behçet's disease. Neurosciences. 2006; 11(4): 260-3. PMid:22266434 